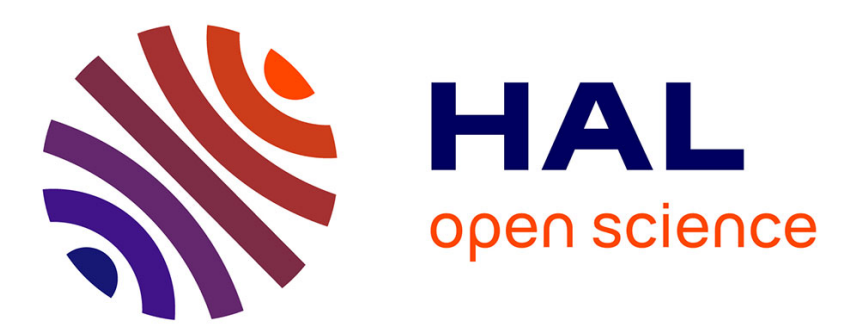

\title{
Yet another computation-oriented necessary and sufficient condition for stabilizability of switched linear systems \\ Mirko Fiacchini
}

\section{- To cite this version:}

Mirko Fiacchini. Yet another computation-oriented necessary and sufficient condition for stabilizability of switched linear systems. IEEE Transactions on Automatic Control, 2022, 67 (7), pp.3627-3632. 10.1109/TAC.2021.3103872 . hal-03246030

\section{HAL Id: hal-03246030 \\ https://hal.univ-grenoble-alpes.fr/hal-03246030}

Submitted on 2 Jun 2021

HAL is a multi-disciplinary open access archive for the deposit and dissemination of scientific research documents, whether they are published or not. The documents may come from teaching and research institutions in France or abroad, or from public or private research centers.
L'archive ouverte pluridisciplinaire HAL, est destinée au dépôt et à la diffusion de documents scientifiques de niveau recherche, publiés ou non, émanant des établissements d'enseignement et de recherche français ou étrangers, des laboratoires publics ou privés. 


\title{
Yet another computation-oriented necessary and sufficient condition for stabilizability of switched linear systems
}

\author{
Mirko Fiacchini
}

\begin{abstract}
This paper presents a computational method to test the stabilizability of discrete-time switched linear systems. The existence of a conic cover of the space on whose elements a convex condition holds is proved to be necessary and sufficient for stabilizability. An algorithm for computing a conic partition that satisfies the new necessary and sufficient condition is given. The algorithm, that allows also to determine bounds on the exponential convergence rate, is proved to overcome the conservatism of conditions equivalent to periodic stabilizability and is applied to a four dimensional system.
\end{abstract}

Index Terms-Switched linear systems, stabilizability, convex analysis.

\section{INTRODUCTION}

Switched systems are characterized by a finite set of possible dynamics, among which the system evolves [14], [21]. In the last decades, this class of systems attracted an increasing attention since it allows to model complex dynamics such as networked and interconnected systems and the interaction between physical systems and digital devices. Classical issues in automatic control, such as stability analysis and control design, result to be rather involved even for switched linear systems, though, yielding to the necessity of a tailored theory [17], [21]. Concerning the problem of stability analysis, several works appeared presenting sufficient and necessary and sufficient conditions. For instance, [18] provides stability analysis based on a variational approach, whereas the work [3] considers mode-dependent Lyapunov functions to provide a sufficient condition for stability.

Concerning the problem of stabilizability of switched linear systems, the necessity of using nonconvex or time-varying functions have been recognized, see [1], and often employed to reduce the conservatism in constructive methods, for instance those based on Lyapunov-Metzler conditions [8], [9], on convex conditions [6], [21], and on quadratic time-varying Lyapunov functions [4]. Also necessary and sufficient conditions for stabilizability appeared, [7], [21], highlighting the inherent complexity of the problem of determining whether a switched linear systems is stabilizable. As a matter of fact, testing whether a necessary and sufficient condition for stabilizability holds is equivalent to check if the unit ball is contained in the interior of the union of a potentially arbitrarily big number of ellipsoids, given by the ball preimages. The stabilizability problem has been proved, indeed, to be undecidable in [13], that addresses the problem in terms of joint spectral subradius, see also [12].

M. Fiacchini is with Univ. Grenoble Alpes, CNRS, Grenoble INP, GIPSAlab, 38000 Grenoble, France. mirko.fiacchiniegipsa-lab.fr.

Research funded in part by ANR via project HANDY, number ANR-18CE40-0010
A strictly weaker condition, referred to as uniform convergence [19], consistent [20], [21] or periodic [6] stabilizability, has been also characterized and its relation with general stabilizability analysed. While [19]-[21] already proved that periodic stabilizability is only sufficient for stabilizability, [6] provides a necessary and sufficient convex condition for periodic stabilizability and its relation with Lyapunov-Metzler conditions. A hint on the conservatism of conditions for periodic stabilizability has been given in [10], expressing it in terms of S-procedure application. Thus, although necessary and sufficient conditions are available, their complexity led to inspect alternative conditions, just sufficient but more computationally affordable. The notable exception [13] proposes algorithms to test necessary and sufficient conditions for stabilizability and to compute tight bounds on the convergence rate.

This paper aims at providing an algorithmic method to test whether a necessary and sufficient condition for stabilizability is satisfied for a switched linear system. The method is based on a novel necessary and sufficient stabilizability condition, consisting in the existence of a conic cover of the space on whose cones a convex condition holds. For switched systems, in fact, conditions based on homogeneous functions and defined on conic partions permit to characterize global properties, see for instance [11], [15], [16]. A major contribution of this paper is the formulation and application of a computational method for generating a sequence of conic covers of the state space for which the new necessary and sufficient condition for stabilizability is satisfied. The method allows also to determine bounds on the exponential convergence rate for a given switching horizon. Although no guarantee for the termination of the procedure can be given, being the problem itself undecidable [13], rather tight numerical results have been obtained. In particular, the method is applied to certify the stabilizability of a system for which the sufficient conditions have been proved not to hold [6]. Moreover, it has been applied to a two dimensional classical example for which tight bounds on the convergence rate are available in the literature [13], showing that analogous estimations are obtained. Finally, a specific four dimensional switched system has been built for which the convergence rate bound can be inferred but hardly computed with alternative necessary and sufficient conditions. The numerical method proved to be able to certify the bound on the convergence rate even for the four dimensional system.

Notation: Given $n \in \mathbb{N}$, define $\mathbb{N}_{n}=\{x \in \mathbb{N}: 1 \leq x \leq n\}$. The subindex denotes, with slight abuse of notation, both the $i$-th row of a matrix (or a vector) and the $i$-th element of a countable set of elements. Given $\Omega \subseteq \mathbb{R}^{n}$, int $(\Omega)$ is the interior of $\Omega$. Given $P \in \mathbb{R}^{n \times n}$ with $P>0$, define $\mathscr{E}(P)=$ $\left\{x \in \mathbb{R}^{n}: x^{T} P x \leq 1\right\}$. The $q$ modes of the switched systems 
are denoted by $\mathscr{I}$, that is $\mathscr{I}=\mathbb{N}_{q}$, then $\mathscr{I}^{k}=\prod_{j=1}^{k} \mathscr{I}$ are all the possible sequences of modes of length $k$ and $\mathscr{I}^{[N]}=\bigcup_{k=1}^{N} \mathscr{I}^{k}$, the sequences of modes of length up to $N$. Given $N \in \mathbb{N}$, the number of elements $i \in \mathscr{I}^{[N]}$ is $\bar{N}=\sum_{k=1}^{N} q^{k}$. Given $i \in \mathscr{I}^{[N]}$ define $\mathbb{A}_{i}=A_{i_{N}} \ldots A_{i_{1}}$ and $\mathscr{B}_{i}=\left\{x \in \mathbb{R}^{n}\right.$ : $\left.x^{T} \mathbb{A}_{i}^{T} \mathbb{A}_{i} x \leq 1\right\}$, with $\mathscr{B}$ unit ball: $\mathscr{B}=\left\{x \in \mathbb{R}^{n}: x^{T} x \leq 1\right\}$. Define $J=\{-1,1\}$ and given $\beta \in J^{n}$, define the matrix $T(\beta) \in \mathbb{R}^{n \times n}$ as $T(\beta)=\operatorname{diag}(\beta)$. The simplex in $\mathbb{R}^{n}$ is denoted as $\Delta^{n}$, that is $\Delta^{n}=\left\{\lambda \in \mathbb{R}^{n}: \lambda \geq 0, \sum_{i=1}^{n} \lambda_{i}=1\right\}$. The sets $\left\{\Gamma_{i}\right\}_{i \in \mathbb{N}_{p}}$ are a cover of $\mathbb{R}^{n}$ if $\bigcup_{i \in \mathbb{N}_{p}} \Gamma_{i}=\mathbb{R}^{n}$.

\section{Problem Statement}

Consider the switched linear system

$$
x_{k+1}=A_{\sigma(k)} x_{k},
$$

where $x_{k} \in \mathbb{R}^{n}$ is the state at time $k \in \mathbb{N}, \sigma: \mathbb{N} \rightarrow \mathscr{I}$ is the switching law and $\left\{A_{i}\right\}_{i \in \mathscr{I}}$, with $A_{i} \in \mathbb{R}^{n \times n}$ for all $i \in \mathscr{I}$, the modes transition matrices. Denote with $x_{k}^{\sigma}(x)$ the state of 11 at time $k$ for a switching law $\sigma(\cdot)$ and an initial condition $x$. The objective is to provide a constructive condition for stabilizability, defined below for the case under analysis.

Definition 1: The system (1) is globally exponentially stabilizable if there are $c \geq 0$ and $\lambda \in[0,1)$ and, for all $x \in \mathbb{R}^{n}$, there exists a switching law $\sigma: \mathbb{N} \rightarrow \mathscr{I}$, such that

$$
\left\|x_{k}^{\sigma}(x)\right\| \leq c \lambda^{k}\|x\|, \quad \forall k \in \mathbb{N}
$$

Since asymptotic and exponential stabilizability are equivalent for this class of systems, there is no loss of generality in considering exponential stabilizability. Necessary and sufficient conditions exist for system (1) to be stabilizable, given first in [21] and in [7] in a set-theoretic version, recalled here.

Theorem $1([7])$ : The switched system $[1]$ is stabilizable if and only if there exists $N \in \mathbb{N}$ such that

$$
\mathscr{B} \subseteq \operatorname{int}\left(\bigcup_{i \in \mathscr{I}[N]} \mathscr{B}_{i}\right)
$$

These results claim substantially that system (1) is stabilizable if and only if a state-dependent switching law exists such that the Euclidean norm of the state can be reduced within the horizon of length $N$. The main problem, that is the objective of the present study, is that checking whether (3) holds might be hardly manageable, for nontrivial cases. Indeed, the problem has been proved to be inherently undecidable, [13].

Other conditions, that are more computationally affordable but just sufficient, have been given in the literature. A first condition is the one based on Lyapunov-Metzler inequalities, [8], [9], posed in terms of bilinear matrix inequalities. Another sufficient condition for stabilizability, in form of convex optimization problem, is given in [6] and recalled hereafter.

Theorem 2 ([6]): The switched system $[1]$ is stabilizable if there exist $N \in \bar{N}$ and $\eta \in \mathbb{R}^{\bar{N}}$ such that $\eta \in \Delta^{\bar{N}}$ and

$$
\sum_{i \in \mathscr{I}^{[N]}} \eta_{i} \mathbb{A}_{i}^{T} \mathbb{A}_{i}<I
$$

Condition (4) has been proved in [6] to be equivalent to periodic stabilizability, also referred to as uniform convergence [19] or consistent stabilizability [20], [21], and less conservative than the Lyapunov-Metzler condition. Moreover, it has been proved in [19], [21] that periodic stabilizability is only sufficient, not necessary for stabilizability. This means that switched linear systems exist such that condition (3) is satisfied with an appropriate $N \in \mathbb{N}$ whereas (4) cannot hold, for every $N \in \mathbb{N}$, nor Lyapunov-Metzler conditions can be satisfied, see the Example 17 in [6] that will be considered in this paper. A hint on the source of conservatism of the condition for periodic stabilizability is given in [10], where condition (4) is proved to be obtainable by resorting to the $\mathrm{S}$-procedure.

The objective of this work is to provide a computational method for obtaining a test for stabilizability which is less conservative than the sufficient ones, in the line of [13], and to show its efficiency even in four dimensions.

\section{NECESSARY AND SUFFICIENT CONDITIONS FOR STABILIZABILITY}

A useful result, whose proof is in [6], is recalled.

Lemma 1 ([6]): Given $P_{i} \geq 0, i \in \mathbb{N}_{m}$, the following equality holds

$$
\bigcup_{\pi \in \Delta^{m}} \mathscr{E}\left(\sum_{i \in \mathbb{N}_{m}} \pi_{i} P_{i}\right)=\bigcup_{i \in \mathbb{N}_{m}} \mathscr{E}\left(P_{i}\right) .
$$

A first novel necessary and sufficient condition for stabilizability is presented below. Based on this condition, another one, more computation oriented, is given afterward.

Theorem 3: The switched system (1) is stabilizable if and only if there exists $N \in \mathbb{N}$ and, for every $x \in \mathbb{R}^{n}$, there is $\eta(x) \in \mathbb{R}^{\bar{N}}$ such that $\eta(x) \in \Delta^{\bar{N}}$ and

$$
x^{T}\left(\sum_{i \in \mathscr{I}[N]} \eta_{i}(x) \mathbb{A}_{i}^{T} \mathbb{A}_{i}\right) x<x^{T} x .
$$

Proof: To prove sufficiency, suppose $\eta(x) \in \Delta^{\bar{N}}$ exists for every $x \in \mathbb{R}^{n}$ such that (5) holds. Condition (5) is equivalent to the fact that if $x$ is in $\mathscr{B}$ then it also belongs to the interior of the set

$$
\mathscr{E}\left(\sum_{i \in \mathscr{I}[N]} \eta_{i}(x) \mathbb{A}_{i}^{T} \mathbb{A}_{i}\right)=\left\{z \in \mathbb{R}^{n}: z^{T}\left(\sum_{i \in \mathscr{I}[N]} \eta_{i}(x) \mathbb{A}_{i}^{T} \mathbb{A}_{i}\right) z \leq 1\right\}
$$

Denoting $\Lambda=\left\{\eta(x): x \in \mathbb{R}^{n}\right\}$, that is a subset of $\Delta^{\bar{N}}$, and from Lemma 1] it follows that

$$
\begin{aligned}
& \mathscr{B} \subseteq \bigcup_{x \in \mathbb{R}^{n}} \operatorname{int}\left(\mathscr{E}\left(\sum_{i \in \mathscr{I}[N]} \eta_{i}(x) \mathbb{A}_{i}^{T} \mathbb{A}_{i}\right)\right) \\
& =\bigcup_{\eta \in \Lambda} \operatorname{int}\left(\mathscr{E}\left(\sum_{i \in \mathscr{I}[N]} \eta_{i} \mathbb{A}_{i}^{T} \mathbb{A}_{i}\right)\right) \subseteq \underset{\eta \in \Delta^{\bar{N}}}{\bigcup} \operatorname{int}\left(\mathscr{E}\left(\sum_{i \in \mathscr{I}[N]} \eta_{i} \mathbb{A}_{i}^{T} \mathbb{A}_{i}\right)\right) \\
& \subseteq \operatorname{int}\left(\bigcup_{\eta \in \Delta^{\bar{N}}} \mathscr{E}\left(\sum_{i \in \mathscr{I}[N]} \eta_{i} \mathbb{A}_{i}^{T} \mathbb{A}_{i}\right)\right)=\operatorname{int}\left(\bigcup_{i \in \mathscr{I}[N]} \mathscr{B}_{i}\right),
\end{aligned}
$$

and then (3) holds. For necessity, suppose that (3) is satisfied. Then every $x \in \mathscr{B}$, i.e. such that $x^{T} x \leq 1$, belongs also to the interior of $\bigcup_{i \in \mathscr{I}[N]} \mathscr{B}_{i}$ which implies, from Lemma 1 and 6 , that $\eta(x) \in \Delta^{N}$ exists such that

$$
x^{T}\left(\sum_{i \in \mathscr{I}[N]} \eta_{i}(x) \mathbb{A}_{i}^{T} \mathbb{A}_{i}\right) x<1 .
$$


Hence, (5) holds.

Theorem 3 substantially claims that the existence of $\eta(x) \in$ $\Delta^{\bar{N}}$ such that 5 is satisfied, for every $x \in \mathbb{R}^{n}$, is necessary and sufficient for the stabilizability of the switched linear system. On the other hand, the determination of such $\eta(x)$ defined on the whole $\mathbb{R}^{n}$ might be computationally intractable. Note that Theorem 3 could have been alternatively proved by noticing that (5) is equivalent to the existence, for every $x \in \mathbb{R}^{n}$ different from the origin, of $i(x) \in \mathscr{I}^{[N]}$, such that $\left\|\mathbb{A}_{i(x)} x\right\|_{2}<\|x\|_{2}$, for appropriate $N \in \mathbb{N}$, and then also equivalent to (2).

Remark 1: The sufficient condition (4) can be seen as the particular case of (5) with $\eta=\eta(x)$ for all $x \in \mathbb{R}^{n}$.

The alternative computation-oriented necessary and sufficient condition can now be given.

Theorem 4: The switched system (1) is stabilizable if and only if there exist: $N \in \mathbb{N}$; a conic cover $\left\{\mathscr{C}^{(j)}\right\}_{j \in \mathbb{N}_{p}}$ of $\mathbb{R}^{n}$; and $\left\{\mu^{(j)}\right\}_{j \in \mathbb{N}_{p}}$ with $\mu^{(j)} \in \Delta^{\bar{N}}$ such that

$$
x^{T}\left(\sum_{i \in \mathscr{I}[N]} \mu_{i}^{(j)} \mathbb{A}_{i}^{T} \mathbb{A}_{i}\right) x<x^{T} x, \quad \forall x \in \mathscr{C}^{(j)} .
$$

Proof: Sufficiency follows directly from Theorem 3 with $\eta_{i}(x)=\mu_{i}^{(j)}$ for all $i \in \mathscr{I}^{[N]}, j \in \mathbb{N}_{p}$, and $x \in \mathscr{C}^{(j)}$. To prove necessity, suppose the system is stabilizable, that is equivalent to satisfaction of (3) for $N \in \mathbb{N}$. Then $\rho>0$ exists such that

$$
\frac{1}{\sqrt{1-\rho}} \mathscr{B} \subseteq \operatorname{int}\left(\bigcup_{i \in \mathscr{I}[N]} \mathscr{B}_{i}\right) \text {. }
$$

Condition (8) implies that for every $x \in \mathbb{R}^{n}$ there exists $\eta(x) \in$ $\mathbb{R}^{\bar{N}}$ such that

$$
x^{T} P(x) x<-\rho x^{T} x
$$

with

$$
P(x)=\sum_{i \in \mathscr{I}[N]} \eta_{i}(x) \mathbb{A}_{i}^{T} \mathbb{A}_{i}-I,
$$

from Theorem 3 . The aim is to prove that 9 implies the existence of the conic covering of $\mathbb{R}^{n}$ and $\mu^{(j)}$ for every cone such that $(7)$ holds. From homogeneity, only $x \in \partial \mathscr{B}$, i.e. with $\|x\|_{2}=1$, are considered in the following. Given $x \in \partial \mathscr{B}$, the function defined for $y \in \mathbb{R}^{n}$ as

$$
f_{x}(y)=(x+y)^{T} P(x)(x+y)-x^{T} P(x) x
$$

is continuous in $y$ and such that $f_{x}(0)=0$, and thus is smaller than $\rho$ for $y$ in a neighborhood of the origin. Then for every $x \in \partial \mathscr{B}$, there exists $\varepsilon(x)$ positive such that $f_{x}(y)<\rho$ for all $y \in \varepsilon(x) \mathscr{B}$. This implies that, taking $\varepsilon^{*}=\min _{x \in \partial \mathscr{B}} \varepsilon(x)$, which exists and is positive, then

$$
(x+y)^{T} P(x)(x+y)=f_{x}(y)+x^{T} P(x) x<0, \quad \forall y \in \varepsilon^{*} \mathscr{B}
$$

for all $x \in \partial \mathscr{B}$, from 9 . Hence, for all $x$ on the boundary of the unit ball, the condition

$$
z^{T}\left(\sum_{i \in \mathscr{I}[N]} \eta_{i}(x) \mathbb{A}_{i}^{T} \mathbb{A}_{i}\right) z<z^{T} z,
$$

holds for all $z$ such that $\|x-z\|_{2} \leq \varepsilon^{*}$, and also for $z$ in the cone generated by $x+\varepsilon \mathscr{B}$, i.e.

$\mathscr{C}_{x}=\left\{y \in \mathbb{R}^{n}: \exists z \in \mathbb{R}^{n}, \exists \lambda \in \mathbb{R}\right.$ s.t. $\left.\|x-z\|_{2} \leq \varepsilon^{*}, y=\lambda z, \lambda \geq 0\right\}$ from homogeneity. Finally, defining: a grid of $x^{(j)} \in \partial \mathscr{B}$, with $j \in \mathbb{N}_{p}$, such that for all $x \in \partial \mathscr{B}$ there is $x^{(j)}$ such that $\| x^{(j)}-$ $x \|_{2} \leq \varepsilon^{*}$; the conic covering $\left\{\mathscr{C}_{x^{(j)}}\right\}_{j \in \mathbb{N}_{p}}$ from $\sqrt{11}$; and $\mu^{(j)}=$ $\eta\left(x^{(i)}\right)$, condition 77 holds.

Theorem 4 means that the existence of a fine enough conic cover of the space such that (7) holds is a necessary and sufficient condition for stabilizability. Moreover, condition (7) boils down to a linear condition if the conic sets $\mathscr{C}^{(j)}$ are defined as the negative sublevel sets of quadratic forms, as will be illustrated below. More details on the computational implication will be given in the following section.

Note that, being the condition given in Theorem 4 necessary and sufficient for stabilizability, it is equivalent to the other ones, [7], [21], and strictly less conservative than sufficient conditions, for instance those based on convex conditions [6], [21], on Lyapunov-Mezler ones [8], [9] and on time-varying Lyapunov functions [4], see [6] for a relation between some of them. Moreover, the satisfaction of the condition in Theorem 4 as for the equivalent ones, implies that the classical min-switch control strategies stabilize the switched system.

\section{CONIC PARTITION AND NONNEGATIVE QUADRATIC FORMS}

The first issue is how to compute a set of quadratic forms determining a cover of the whole space, on whose cones condition (7) has to be tested. That is, the design of a set of matrices $\left\{M_{j}\right\}_{j \in \mathbb{N}_{p}}$ such that

$$
\bigcup_{j \in \mathbb{N}_{p}}\left\{x \in \mathbb{R}^{n}: x^{T} M_{j} x \geq 0\right\}=\mathbb{R}^{n} .
$$

Moreover, it might be necessary to iteratively generate finer covers of the state space.

The presented method is based on generating covers of the state space composed by the closed convex cones determined by a basis matrix. Hence, for every cone, a quadratic form that is nonnegative on the cone is defined. Finally a method to generate finer covers of the state space, by replacing a cone with a conic cover of it, is given.

\section{A. Initial conic partition generation}

Any basis of the state space is the linear transformation of the standard basis of $\mathbb{R}^{n}$, denoted $\left\{e_{i}\right\}_{i \in \mathbb{N}_{n}}$. Indeed, clearly, the elements of any given basis $\left\{b_{i}\right\}_{i \in \mathbb{N}_{n}}$ with $b_{i} \in \mathbb{R}^{n}$ are $B e_{i}=\left[\begin{array}{lllll}b_{1} & b_{2} & \ldots & b_{n}\end{array}\right] e_{i}=b_{i}$, for all $i \in \mathbb{N}_{n}$. Moreover, given a nonsingular matrix $B \in \mathbb{R}^{n \times n}$ the set

$$
C(B)=\left\{x \in \mathbb{R}^{n} \mid \exists \lambda \in \mathbb{R}^{n}: B \lambda=x, \lambda \geq 0\right\}
$$

is a closed convex cone. Then given the basis $\left\{b_{i}\right\}_{i \in \mathbb{R}^{n}}$ of $\mathbb{R}^{n}$, the closed convex cones $C(B T(\beta))$ defined for all $\beta \in J^{n}$, are such that $\bigcup_{\beta \in I^{n}} C(B T(\beta))=\mathbb{R}^{n}$. Thus, every nonsingular matrix $B$ defines a conic partition of $\mathbb{R}^{n}$, that will be used in the algorithm as initial partition.

\section{B. Nonnegative quadratic form}

Given $\beta \in J^{n}$ and the cone $C(B T(\beta))$, a quadratic form that is nonnegative on $C(B T(\beta))$ must be defined. Given $\beta \in J^{n}$, denote $V=B T(\beta)$ in this section, to ease to notation. 
Lemma 2: Given $\left\{v_{i}\right\}_{i \in \mathbb{N}_{n}}$ basis of $\mathbb{R}^{n}$ and $V=\left[\begin{array}{llll}v_{1} & v_{2} & \ldots & v_{n}\end{array}\right]$, if $M(V) \in \mathbb{R}^{n \times n}$ symmetric satisfies

$$
v_{i}^{T} M(V) v_{j} \geq 0, \quad \forall i, j \in \mathbb{N}_{n} \text { s.t. } i \leq j
$$

then $x^{T} M(V) x \geq 0$ for all $x \in C(V)$.

Proof: By definition of the convex cone $C(V)$, for every $x \in C(V)$ there exists $\lambda \in \mathbb{R}^{n}$ with $\lambda \geq 0$ such that $x=\sum_{i=1}^{n} \lambda_{i} v_{i}$. Then $x^{T} M(V) x$ is given by a finite sum of terms $\lambda_{i} \lambda_{j} v_{i} M(V) v_{j}$, that are nonnegative from 14 . Hence $x^{T} M(V) x \geq 0$ for all $x \in C(V)$.

Lemma 2 can be used to determine a quadratic form that is nonnegative on the cone generated by every $\left\{v_{i}\right\}_{i \in \mathbb{N}_{n}}$ basis of $\mathbb{R}^{n}$. Recall that $M(V)$ might not be positive definite to determine nontrivial cones.

Proposition 1: Given $\left\{v_{i}\right\}_{i \in \mathbb{N}_{n}}$ basis of $\mathbb{R}^{n}$ and $V=$ $\left[\begin{array}{llll}v_{1} & v_{2} & \ldots & v_{n}\end{array}\right]$ the following inclusion holds

$$
C(V) \cup C(-V) \subseteq\left\{x \in \mathbb{R}^{n}: x^{T} M(V) x \geq 0\right\}
$$

for all $M(V)$ satisfying (14).

Proof: The fact that $C(V) \subseteq\left\{x \in \mathbb{R}^{n}: x^{T} M(V) x \geq 0\right\}$ follows directly from Lemma 2 Moreover, if $M(V)$ satisfies the conditions in (14), than it also satisfies them for $\left\{-v_{i}\right\}_{i \in \mathbb{N}_{n}}$, which implies that also $C(-V) \subseteq\left\{x \in \mathbb{R}^{n}: x^{T} M(V) x \geq 0\right\}$.

An implication of Proposition 1 is that some of the sets in the cover $\bigcup_{\beta \in I^{n}} C(B T(\beta))$ could be disregarded from the analysis, since redundant.

\section{Finer partition generation}

As it will be clearer from the following section, a method is necessary to split a closed convex cone $C(V)$ in smaller cones whose union contains $C(V)$. In particular, given the nonsingular matrix $V$, a first method is provided for determining $n$ nonsingular matrices $U_{j}$, with $j \in \mathbb{N}_{n}$ such that

$$
C(V)=\bigcup_{j \in \mathbb{N}_{n}} C\left(U_{j}\right) .
$$

The simpler approach proposed consists basically in using the barycenter vector $\bar{v}(V)=1 / n \sum_{j \in \mathbb{N}_{n}} v_{j}$ to split the cone:

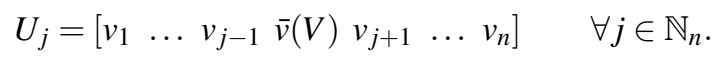

As $\bar{v}(V)$ belongs to the convex hull of $\left\{v_{i}\right\}_{i \in \mathbb{N}_{n}}$ and matrices $U_{j}$ are nonsingular, for every $j \in \mathbb{N}_{n}$, then their columns are basis of $\mathbb{R}^{n}$ and satisfy (16). This means that, given a conic partition of $\mathbb{R}^{n}$, another partition of $\mathbb{R}^{n}$ is obtained by replacing the cone $C(V)$ with the cones $C\left(U_{j}\right)$ as in 177 .

Alternatively, a cone can be splitted along the longer edge of the set $\operatorname{co}\left(\left\{v_{j}\right\}_{j \in \mathbb{N}_{n}}\right)$. This consists in defining

$$
\begin{aligned}
& \left(i^{*}, j^{*}\right)(V) \in \arg \max _{(i, j) \in \mathbb{N}_{n}^{2}}\left\|v_{i}-v_{j}\right\|, \\
& v^{*}(V)=0.5\left(v_{i^{*}}+v_{j^{*}}\right)
\end{aligned}
$$

to split the cone in two

$$
\begin{aligned}
& U_{i^{*}}=\left[\begin{array}{lllllll}
v_{1} & \ldots & v_{i^{*}-1} & v^{*}(V) & v_{i^{*}+1} & \ldots & v_{n}
\end{array}\right], \\
& U_{j^{*}}=\left[\begin{array}{lllllll}
v_{1} & \ldots & v_{j^{*}-1} & v^{*}(V) & v_{j^{*}+1} & \ldots & v_{n}
\end{array}\right] .
\end{aligned}
$$

Also in this case matrices $U_{i^{*}}$ and $U_{j^{*}}$ are nonsingular and satisfy $C(V)=C\left(U_{i^{*}}\right) \cup C\left(U_{j^{*}}\right)$. Moreover, the maximal distances between the vectors $v_{j}$ determining a partition would converge to zero by iterating the splitting procedure.

\section{LOCAL CONTRACTION CONSTRAINT AND ALGORITHM}

Now that a conic partition is given and a quadratic form that is nonnegative on every cone can be determined, the contraction conditions can be posed over every cone. Suppose that a set of basis $\mathscr{V}=\left\{V^{(j)}\right\}_{j \in \mathbb{N}_{p}}$ determining the cover of $\mathbb{R}^{n}$ as $\bigcup_{j \in \mathbb{N}_{p}} C\left(V^{(j)}\right)$ has been obtained as illustrated above, i.e. starting with an initial cover and then refining it if necessary. Also the following set of cones

$$
\bigcup_{j \in \mathbb{N}_{p}}\left\{x \in \mathbb{R}^{n}: x^{T} M\left(V^{(j)}\right) x \geq 0\right\}
$$

is a cover of $\mathbb{R}^{n}$, from Proposition 1 .

Proposition 2: Given $\left\{v_{i}\right\}_{i \in \mathbb{N}_{p}}$ basis of $\mathbb{R}^{n}$ and $V=$ $\left[\begin{array}{llll}v_{1} & v_{2} & \ldots & v_{n}\end{array}\right]$, the condition $(7]^{2}$ is satisfied with $\mathscr{C}^{(j)}=$ $C(V) \cup C(-V)$ and $\mu^{(j)}=\mu(V)$ if

$$
\sum_{i \in \mathscr{I}[N]} \mu_{i}(V) \mathbb{A}_{i}^{T} \mathbb{A}_{i}-I+M(V)<0,
$$

holds with $M(V)$ satisfying 14 .

Proof: From Proposition 1, condition (7) with $\mathscr{C}^{(j)}=$ $C(V) \cup C(-V)$ and $\mu^{(j)}=\mu(V)$ holds if

$$
x^{T}\left(\sum_{i \in \mathscr{I}[N]} \mu_{i}(V) \mathbb{A}_{i}^{T} \mathbb{A}_{i}\right) x<x^{T} x, \quad \forall x \text { s.t. } x^{T} M(V) x \geq 0
$$

is satisfied, and then also if (20) holds, from S-procedure [2]. Thus, condition 20 implies satisfaction of 7 with $\mu^{(j)}=$ $\mu(V)$ and $\mathscr{C}^{(j)}=C(V) \cup C(-V)$.

The interest of this result lies in the fact that 20 is more suitable than (7) from the computational point of view.

Finally, a sketch of algorithm is given for testing whether the condition in Theorem 4 is satisfied and hence if the system (11) is stabilizable, see Algorithm 11. If Algorithm 1 terminates, then the system is stabilizable.

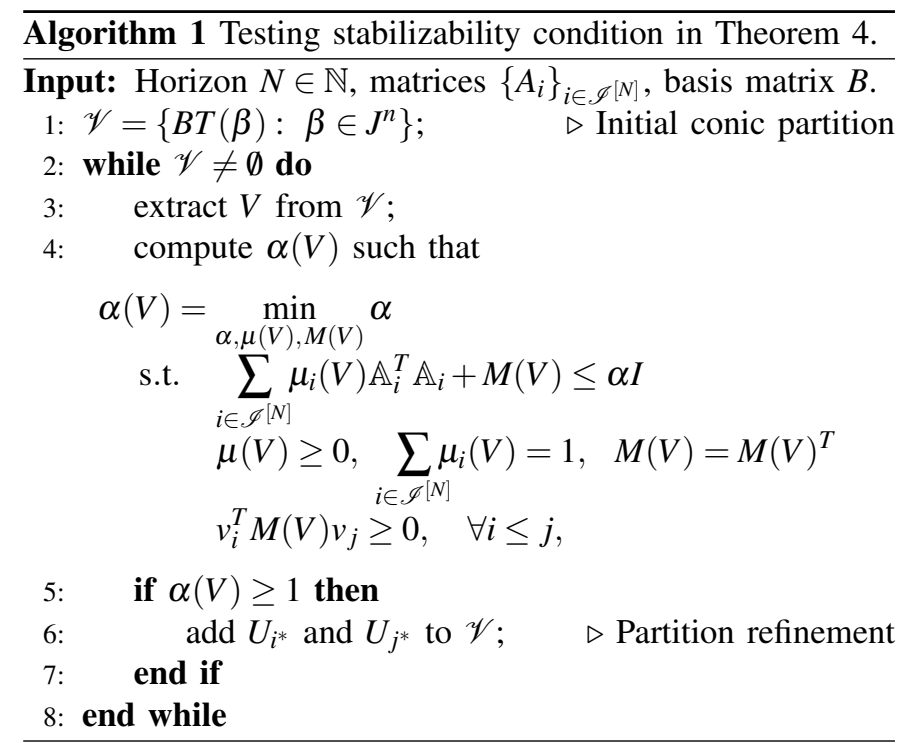

Remark 2: The conditions presented in this paper as well as the Algorithm 1 can be adapted to determine, besides the property of stabilizability, bounds on the exponential convergence rate $\lambda$, sometimes referred to as stabilization radius [13]. It is sufficient, indeed, to multiply all the matrices 


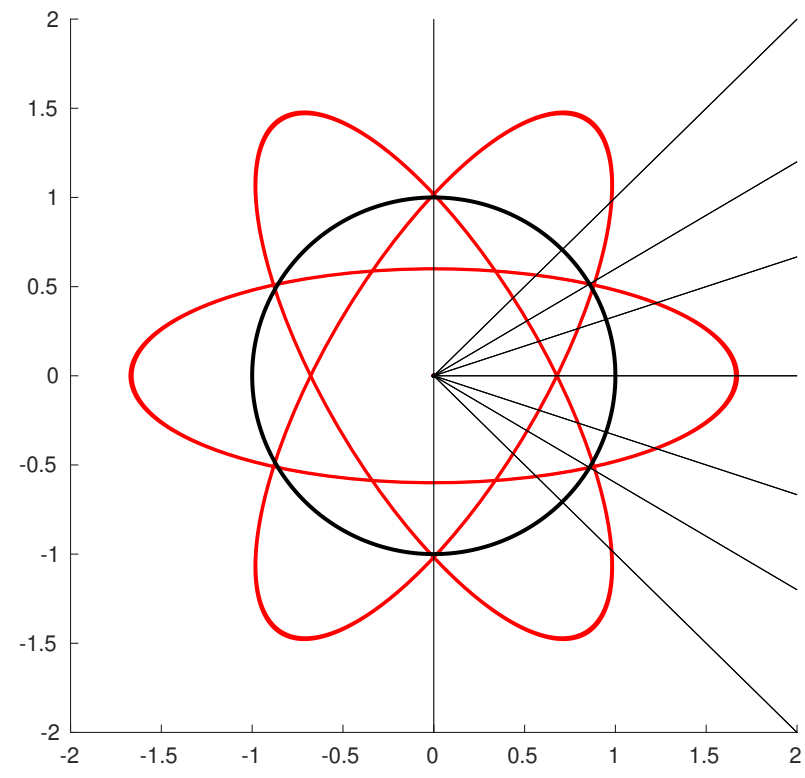

Fig. 1. Set $\mathscr{B}$, black, ellipsoidal preimages $\mathscr{B}_{1}, \mathscr{B}_{2}$ and $\mathscr{B}_{3}$, in red, and basis vectors of $\left\{V^{(j)}\right\}_{j \in \mathbb{N}_{8}}$, in thin lines.

by $1 / \lambda$ in the stabilizability conditions. If the condition holds, then the system has a convergence rate bounded above by $\lambda$, see Examples 2 and 3 below.

A convex problem, in LMI form, has to be solved at every iteration. On the other hand, there is no guarantee, in general, of finite termination of Algorithm 1 , as the existence of a conic partition cannot be ensured unless the system is stabilizable. This is reasonable since the problem is undecidable by its nature. Moreover, the number of elements in an eventual conic partition satisfying the necessary and sufficient condition could be arbitrarily big. Nonetheless, as illustrated in the next section, the application of Algorithm 1 to examples from the literature and to a four dimensional system shows the efficiency of the algorithm in testing stabilizability and computing tight bounds on the exponential convergence rate.

\section{NUMERICAL EXAMPLES}

Three examples will be considered to illustrate the results and the application of the algorithm. The set of matrices $M(V)$, determining the conic cover, and the parameters $\alpha(V)$ and $\mu(V)$ resulting from the application of Algorithm 1 for each example are available at [5], in form of matlab data files, together with simple scripts to verify the conic covering of the space and the stabilizability condition (20) satisfaction.

Example 1: Consider Example 17 in [6], employed to prove that condition (4) is just sufficient for stabilizability. The system is given by three modes with matrices

$$
A_{1}=A R(0), \quad A_{2}=A R\left(\frac{2 \pi}{3}\right), \quad A_{3}=A R\left(\frac{-2 \pi}{3}\right),
$$

where

$$
A=\left[\begin{array}{cc}
0.6 & 0 \\
0 & 0.6^{-1}
\end{array}\right], \quad R(\theta)=\left[\begin{array}{cc}
\cos (\theta) & -\sin (\theta) \\
\sin (\theta) & \cos (\theta)
\end{array}\right] .
$$

The system is proved in [6] to be stabilizable since the necessary and sufficient condition (3) is satisfied with $N=1$.

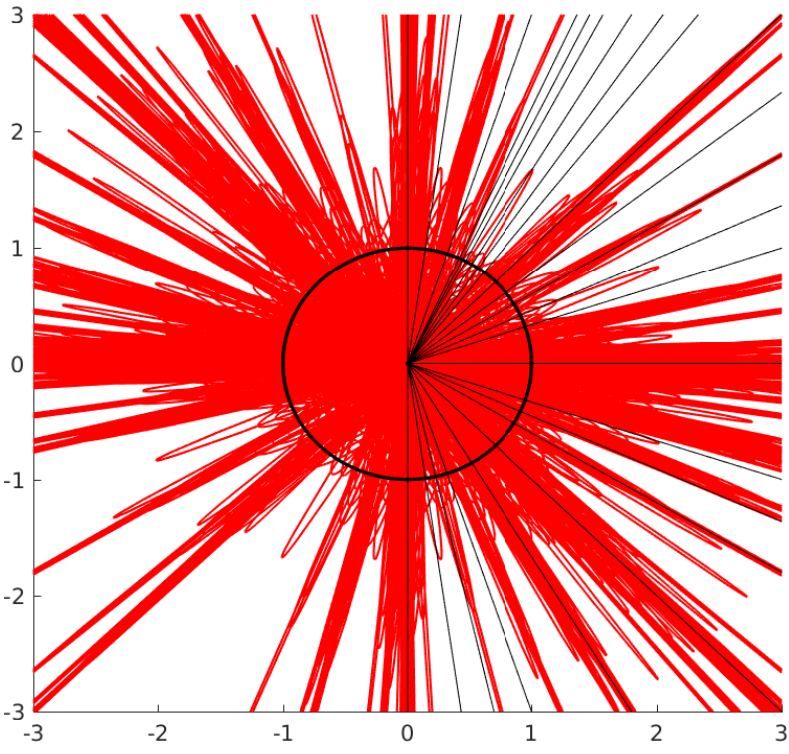

Fig. 2. Set $\mathscr{B}$, black, ellipsoidal preimages $\mathscr{B}_{i}$ for $i \in \mathscr{I}^{[9]}$, in red, and basis vectors of $\left\{V^{(j)}\right\}_{j \in \mathbb{N}_{23}}$, in black lines.

Then, the Euclidean norm decreases at every step, but (4) does not hold, for any $N \in \mathbb{N}$. This implies also that LyapunovMetzler conditions cannot validate stabilizability either.

Algorithm 1 with $N=1$ and the identity as initial basis matrix, i.e. $B=I$, has been applied to this example, validating the stabilizability with a final cover of 8 cones $\left\{V^{(j)}\right\}_{j \in \mathbb{N}_{8}}$, depicted in Figure 1 Figure 1 represents also the sets $\mathscr{B}$ and its preimages $\mathscr{B}_{1}, \mathscr{B}_{2}$ and $\mathscr{B}_{3}$ that graphically validate condition (3) with $N=1$. Note that from Proposition 11, it is sufficient to generate a cover of half of the space $\mathbb{R}^{2}$.

Example 2: The second switched system, with modes

$$
A_{1}=R\left(\frac{\pi}{4}\right), \quad A_{2}=\left[\begin{array}{cc}
2 & 0 \\
0 & 1 / 2
\end{array}\right]
$$

is based on an example from [19] and used in [13] to illustrate methods for computing bounds of the stabilizability radius. These methods, based on conditions evaluated on a grid of the unit circle in $\mathbb{R}^{2}$, hence on a one dimensional space, provides two bound estimations, of 0.886 and 0.88 .

To infer the value of the convergence rate $\lambda$, consider the system whose matrices are given by $\bar{A}_{1}=\lambda^{-1} A_{1}$ and $\bar{A}_{2}=$ $\lambda^{-1} A_{2}$. For $N=9$ and $\lambda=0.8855$ the set $\mathscr{B}$ and the preimages $\mathscr{B}_{i}$ for all $\mathscr{I}^{[9]}$, whose number is $\bar{N}=1022$, are drawn, see Figure 2. From geometric inspection it can be proved that $\mathscr{B}$ is contained in the union of ellipsoids and $\lambda=0.8855$ seems a rather tight bound for $N=9$, see the Figure 3

Algorithm 1 is then applied with $B=I$, validating the condition of stabilizability with convergence rate of $\lambda=0.8855$ by testing the convex condition over 23 cones covering the half space, also depicted in Figure 2

Comparing this result with those obtained in [13] it is worth noting that, besides providing a tighter bound with respect to one proposed, the method presented here provides guarantee of stabilizability, that cannot be ensured in general when evaluating conditions on a grid of points. Another benefit is the fact that, as the proposed method does not resort to a grid 


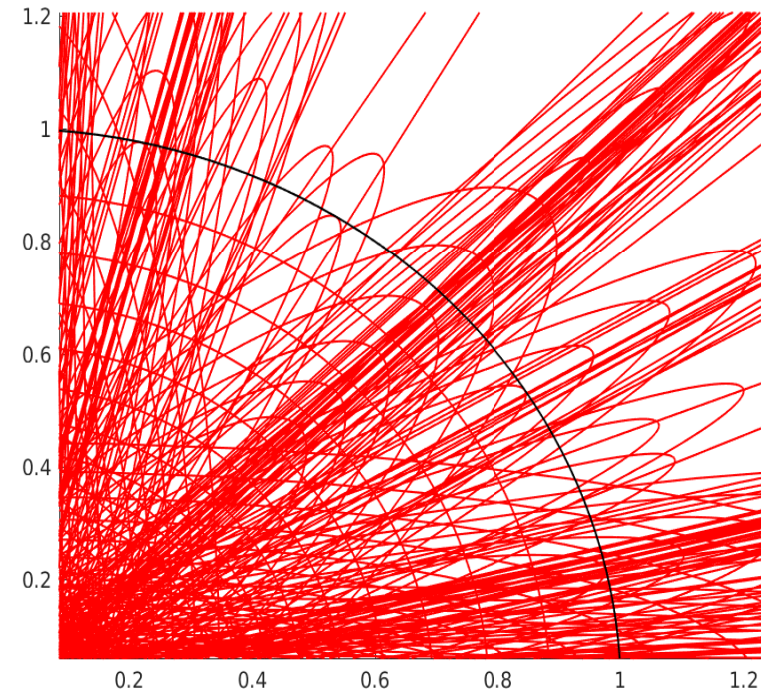

Fig. 3. Zoom of set $\mathscr{B}$, black, ellipsoidal preimages $\mathscr{B}_{i}$ for $i \in \mathscr{I}^{[9]}$, in red, and basis vectors of $\left\{V^{(j)}\right\}_{j \in \mathbb{N}_{23}}$.

of the space, it can be applied to higher dimensional systems, as illustrated in the next example. For this, the algorithm has also been applied to this example with $N=5$, leading to the validation of a bound of $\lambda=0.96$ with 10 cones.

Example 3: This example is specifically built such that a tight estimate of the convergence rate is known but other methods for its sharp estimation, based on the space gridding, might be not applicable due to the space dimension. To illustrate the possibility of applying the proposed method in higher dimension, in fact, a system is considered that is composed by two subsystems equal to the one of Example 2. Any method based on gridding the space is hardly applicable in $\mathbb{R}^{4}$. Moreover, an accurate estimation of the convergence rate is known by construction, and then the precision of the estimation obtained with Algorithm 1 can be objectively evaluated. Consider the four dimensional system with 4 modes

$$
\begin{aligned}
\hat{A}_{1}=\left[\begin{array}{cc}
\lambda^{-1} A_{1} & 0 \\
0 & \delta I
\end{array}\right], & \hat{A}_{2}=\left[\begin{array}{cc}
\lambda^{-1} A_{2} & 0 \\
0 & \delta I
\end{array}\right], \\
\hat{A}_{3}=\left[\begin{array}{cc}
\delta I & 0 \\
0 & \lambda^{-1} A_{1}
\end{array}\right], & \hat{A}_{4}=\left[\begin{array}{cc}
\delta I & 0 \\
0 & \lambda^{-1} A_{2}
\end{array}\right],
\end{aligned}
$$

with $\lambda$ the known estimation of the convergence rate, see below, and $A_{1}$ and $A_{2}$ as in 22). Parameter $\delta$ is posed equal to 0.999 , to guarantee a decreasing of the function $x^{T} x$ within the horizon $N$ of the four dimensional system. The objective is to check whether Algorithm 1 is able to validate the convergence rate for the four dimensional system.

Since $N=9$, with $q=4$, leads to more than a half million of sequences, the smaller horizon of $N=5$ has been employed. Note that $N=5$ gives $\bar{N}=1364$ switching elements in $\mathscr{I}^{[5]}$. Since the bound of $\lambda=0.96$ has been validated in the previous example for the single subsystem, it must hold also for the four dimensional system. The objective is to test whether the tight bound $\lambda=0.96$ for $N=5$ can be validated also in this case.

Indeed, the application of Algorithm 1, with a randomly generated non-singular matrix $B$, provides a validation of the bound $\lambda=0.96$ for the four dimensional system, the semidefinite conditions holding in each of the 1827 cones composing the generated cover of $\mathbb{R}^{4}$.

\section{CONCLUSIONS}

In this paper a novel necessary and sufficient condition for stabilizability has been proposed. The condition consists in the existence of an appropriate conic cover of the space and a convex condition on its elements. This condition led to a procedure for iteratively generating a sequence of conic covers and linear problems allowing to test the stabilizability of systems for which no periodic stabilizability can hold. The method has been also applied to estimate the convergence rate for switched linear systems, even in dimension four.

\section{REFERENCES}

[1] F. Blanchini and C. Savorgnan. Stabilizability of switched linear systems does not imply the existence of convex Lyapunov functions. Automatica, $44: 1166$ - 1170, 2008.

[2] S. Boyd, L. El Ghaoui, E. Feron, and V. Balakrishnan. Linear Matrix Inequalities in system and control theory. SIAM, 1994.

[3] J. Daafouz, P. Riedinger, and C. Iung. Stability analysis and control synthesis for switched systems : A switched Lyapunov function approach. IEEE Transactions on Automatic Control, 47:1883-1887, 2002.

[4] G. S. Deaecto and J. C. Geromel. Stability and performance of discretetime switched linear systems. Systems \& Control Letters, 118:1-7, 2018.

[5] M. Fiacchini. http://www.gipsa-lab.fr/ mirko.fiacchini/NSCswitch

[6] M. Fiacchini, A. Girard, and M. Jungers. On the stabilizability of discrete-time switched linear systems: novel conditions and comparisons. IEEE Transactions on Automatic Control, 61(5):1181-1193, 2016.

[7] M. Fiacchini and M. Jungers. Necessary and sufficient condition for stabilizability of discrete-time linear switched systems: A set-theory approach. Automatica, 50(1):75 - 83, 2014.

[8] J. C. Geromel and P. Colaneri. Stability and stabilization of continuoustime switched linear systems. SIAM J. Control Optim., 45(5):19151930, 2006.

[9] J. C. Geromel and P. Colaneri. Stability and stabilization of discretetime switched systems. International Journal of Control, 79(7):719-728, July 2006.

[10] W. P. M. H. Heemels, A. Kundu, and J. Daafouz. On Lyapunov-Metzler inequalities and S-procedure characterizations for the stabilization of switched linear systems. IEEE Transactions on Automatic Control, 62(9):4593-4597, 2016

[11] R. Iervolino, F. Vasca, and L. Iannelli. Cone-copositive piecewise quadratic Lyapunov functions for conewise linear systems. IEEE Transactions on Automatic Control, 60(11):3077-3082, 2015.

[12] R. M. Jungers. The Joint Spectral Radius: Theory and Applications. Springer-Verlag., Berlin Heidelberg, June 2009.

[13] R. M. Jungers and P. Mason. On feedback stabilization of linear switched systems via switching signal control. SIAM Journal on Control and Optimization, 55(2):1179-1198, 2017.

[14] D. Liberzon. Switching in Systems and Control. Birkhäuser, Boston, MA, 2003.

[15] H. Lin and P. J. Antsaklis. Synthesis of uniformly ultimate boundedness switching laws for discrete-time uncertain switched linear systems. In 42nd IEEE International Conference on Decision and Control, volume 5, pages 4806-4811. IEEE, 2003.

[16] H. Lin and P. J. Antsaklis. Switching stabilizability for continuoustime uncertain switched linear systems. IEEE Transactions on automatic control, 52(4):633-646, 2007.

[17] $\overline{\text { H. Lin }}$ and P. J. Antsaklis. Stability and stabilizability of switched linear systems: a survey of recent results. IEEE Transaction on Automatic Control, 54(2):308-322, 2009.

[18] M. Margaliot. Stability analysis of switched systems using variational principles: An introduction. Automatica, 42:2059-2077, 2006.

[19] D. P. Stanford and J. M. Urbano. Some convergence properties of matrix sets. SIAM Journal on Matrix Analysis and Applications, 15(4):1132 $1140,1994$.

[20] Z. Sun. Stabilizability and insensitivity of switched linear systems. IEEE Transactions on Automatic Control, 49(7):1133-1137, 2004.

[21] Z. Sun and S. S. Ge. Stability Theory of Switched Dynamical Systems. Springer, 2011. 